finden. Ich jedenfalls bin nach einschlägigen Erfahrungen als »Hilfsbremser der 401 Gerechtigkeit « bei aller juristischen Unkenntnis nicht mehr laienhaft genug, um Ihren Zwecken zu genügen.

Mit vorzüglicher Hochachtung (gez. Heidewig Fankhänel)

\title{
Sozialarbeit trotz Gesetz
}

I. KLEINE ANFRAGE DES ABG. BORSCHE (CDU) VOM 24. 9. 1974 IM HESSISCHEN LANDTAG

betreffend Vorlesungen im Fachbereich Sozialarbeit an der Fachhochschule Frankfurt

\section{Ich frage die Landesregierung:}

1. Ist der Landesregierung bekannt, daß im Fachbereich Sozialarbeit an der Fachhochschule Frankfurt sowohl im Fach Jugendhilfe als auch im Fach Berufsfeldanalyse Lehrveranstaltungen unter dem Thema "Sozialarbeit trotz Gesetz « für das Wintersemester I 974/75 angekündigt werden?

2. Hält es die Landesregierung für angebracht, daß - wie diese Ankündigung erkennen läßt - in einer staatlichen Fachhochschule von einem Landesbediensteten Lehrveranstaltungen durchgeführt werden, die offensichtlich eine Art der Sozialarbeit darstellen sollen, die außerhalb des gesetzlichen Rahmens liegen soll?

Wenn nein: Wird die Landesregierung Konsequenzen aus diesem Vorgang ziehen? Welche?

\section{ANTWORT DES FACHBEREICHS SOZIALARBEIT DER FACH- HOCHSCHULE FRANKFURT/M. VOM 2 I. 10. 1974}

$\mathrm{Zu}$ der Kleinen Anfrage des Abgeordneten Borsche (CDU) vom 24.9. 1974 nimmt die Fachbereichskonferenz des Fachbereichs Sozialarbeit der Fachhochschule Frankfurt am Main aufgrund einstimmigen Beschlusses vom 1 5. 10. 1974 wie folgt Stellung:

1. Der Abg. Borsche verdächtigt einen Kollegen, in seinen Lehrveranstaltungen »eine Art der Sozialarbeit dar(zu)stellen«, »die außerhalb des gesetzlichen Rahmens liegen soll. « Dieser Verdächtigung liegt - nimmt man sie beim Wort - die Vorstellung zugrunde, rechtmäßige Sozialarbeit könne in der Bundesrepublik Deutschland nur auf Grund und im Rahmen von Gesetzen geschehen. Eine solche Vorstellung ist bar jeder Sachkenntnis. Dem Bildungsexperten Borsche seien folgende Tatsachen in Erinnerung gerufen:

a) Weite Bereiche der Sozialarbeit werden noch immer yon privaten Trägern verwaltet. Diese privaten Träger haben sich bisher meist erfolgreich gegen eine Bindung ihrer Arbeit an Gesetze gewehrt mit der Folge, daß ein erheblicher Teil ihrer Sozialarbeit zwar unter Verwendung von öffentlichen Steuergeldern, aber "außerhalb des gesetzlichen Rahmens und ohne parlamentarische Kontrolle geschieht.

Tatkräftig unterstützt werden die privaten Träger bei ihrer Sozialarbeit 
"außerhalb des gesetzlichen Rahmens « von der Partei des Abg. Borsche. Entweder mit Sprüchen wie:

"Martin Luther wendet sich in diesen Fragen, von denen Herr von Bodelschwingh so schön gesprochen hat, überhaupt gegen eine öffentliche Zuständigkeıt. «

(So Dr. Barzel (CDU/CSU) in: Verhandlungen des Deutschen Bundestages, 3. Wahlperiode, Stenographische Berichte, Band 49, S. 9039).

Oder mit Gesetzesbestimmungen wie $\$ 10$ BSHG, dessen Abs. 2 Satz 2

"betont .. die Selbständigkeıt der freien Wohlfahrtspflege und damit deren Unabhängıg. kett von den Bestımmungen des BSHG."

(So die Begründung der von der CDU/CSU geführten Bundesregierung zu dem von ihr vorgelegten Entwurf eines BSHG, Verhandlungen des Deutschen Bundestages, 3. Wahlperiode, Drucksache I 799, S. 39).

b) Aber auch soweit Sozialarbeit staatlich organisiert ist, bewegt sie sich nicht selten "außerhalb des gesetzlichen Rahmens «. Hingewiesen sei hier nur auf den Strafvollzug, der alles andere, nur nicht durch oder aufgrund eines Gesetzes geregelt ist. (Vgl. Beschluß des BVerfG vom 1 4. 3. 1972, BVerfGE 33, 1 ff.).

$\mathrm{Da}$ demnach Sozialarbeit "außerhalb des gesetzlichen Rahmens " durchaus üblich ist, kommt der Fachgereich Sozialarbeit nicht umhin, in Lehrveranstaltungen diese Art der Sozialarbeit darzustellen. Keinem Kollegen, der solche Lehrveranstaltungen anbietet, kann daraus ein Vorwurf erwachsen. Der Vorwurf: Sozialarbeit »außerhalb des gesetzlichen Rahmens « trifft - wenn ïberhaupt jemanden - dann den Gesetzgeber, d. h. unter anderen den Abg. Borsche selbst.

2. Damit die Kleine Anfrage - jedenfalls für den Abg. Borsche - überhaupt einen Sinn bekommt, muß man aus ihr wohl die Unterstellung herauslesen, der von der Anfrage betroffene Kollege propagiere in seinen Lehrveranstaltungen eine Sozialarbeit auch "gegen « das Gesetz. Das ist nicht der Fall.

Der betroffene Kollege behandelt in seinen Lehrveranstaltungen allerdings das - gerade Sozialarbeiter bedrängende - Problem des Verhältnisses von Gesetz und gesellschaftlicher Entwicklung. Dieses Problem ist nicht neu. Dem Bildungsexperten und Frankfurter Bürger Borsche ist sicher bekannt, daß ein berïhmter Frankfurter Jurist dieses Problem mit den Worten zu beschreiben versucht hat:

"Es erben sich Gesetz und Rechte wie ene ewige Krankheit fort.

Sie schleppen von Geschlecht sıch zu Geschlechte und rücken sacht von Ort zu Ort.

Vernunft wird Unsinn - Wohltat Plage.

Weh dir, daß du ein Enkel bist.

Die hier beschriebene Rückständigkeit von Gesetzen ist es, die Sozialarbeiter bedrückt. So stehen beispielsweise Sozialarbeiter in der Jugendhilfe täglich vor der Frage, wie sie sinnvoll arbeiten sollen trotz einer

"Gesetzgebung, (die) mit dem Streben junger Menschen nach Emanzipation und Mitverantwortung nicht Schritt gehalten hat."

(So Käte Strobel, Bundesministerin für Jugend, Familie und Gesundheit in: Dritter Jugendbericht, Bonn 1972 , Vorwort.)

Diese Frage muß in der Ausbildung von Sozialarbeitern nicht nur gestellt werden, es müssen auch Wege diskutiert werden, die aus diesem, vom Gesetzgeber mitproduzierten Dilemma herausführen. Dabei bieten sich viele Wege an, die ohne den vom Abg. Borsche unterstellten Bruch von Gesetzen auskommen. 
Zum Beweis dafür, daß die Verdächtigung, der betroffene Kollege propagiere den Gesetzesbruch, aus der Luft gegriffen ist, wird in der Anlage ein Diskussionspapier beigefügt, das der betroffene Kollege verfaßt hat. Dieses Papier zum Thema "Ferienpädagogik und Recht « hat er auf einer Fortbildungstagung der Stadt Offenbach am 6. und 7. I0. 1973 an ca. 70 Jugendleiter verteilt und mit diesen diskutiert. In diesem Papier weist er wiederholt darauf hin, daß der Sozialarbeiter um das geltende Recht nicht herumkommt.

Die Einleitung dieses Papiers stellt übrigens die Zusammenfassung einer I 970 erschienenen, vom Landesjugendring Berlin verlegten und vom »Bund Deutscher Pfadfinder * vertriebenen Broschüre dar. Sie trägt den Titel »Erziehung trotz Gesetz «. Der betroffene Kollege hat diesen Titel leicht abgewandelt für seine Lehrveranstaltungen übernommen, weil dieser Titel die angehenden Sozialarbeiter zum Nachdenken und Diskutieren anregt.

3. Uber die unmittelbare Beantwortung der Anfrage hinausgehend sei festgestellt, daß sich die Frage von Gesetzestreue nicht nur dann stellt, wenn reaktionäre Gesetze Sozialarbeiter an vernünftiger Arbeit hindern. Auch der umgekehrte Fall, daß ein verhältnismäßig fortschrittliches Sozialarbeitsgesetz von Regierungsspitzen systematisch mißachtet wird, ist in der Bundesrepublik Deutschland nicht ausgeschlossen. Diese Behauptung läßt sich beispielsweise belegen an der Geschichte des niedersächsischen Jugendarbeitsschutzgesetzes von 1948, die Gegenstand einer Lehrveranstaltung des von der Anfrage betroffenen Kollegen im Sommersemester 1974 war. Dieses Gesetz sah den Schutz von Lehrlingen und Jungarbeitern in viel größerem Umfang vor als alle früheren und späteren Jugendarbeitsschutzgesetze. Deshalb ist das Gesetz auch von fast allen Unternehmern nicht eingehalten worden. Aber nicht nur die Unternehmer, auch die Landesregierung hat sich an das Gesetz nicht gebunden gefühlt.

"Seit dem 1. September 1949 1st das Gesetz voll in Kraft, aber es wird nurgends durchgeführt. Die Landesregierung hat darïber hinaus sogar einen Beschluß gefaßt, wonach Anzeıgen wegen Ubertretung des Gesetzes nach diesem Paragraphen als Bagatellfälle niedergeschlagen werden sollen «

(So Bundesminister für Arbeit Storch in: Verhandlungen des Deutschen Bundestages, I. Wahlperiode, Stenographische Berichte, S. 4312)

Der damalige niedersächsische Arbeitsminister Kubel und heutige niedersächsische Ministerpräsident erklärte vor dem Landtag:

"Ich habe starke Hemmungen gehabt, das vom Landtag beschlossene ursprüngliche Gesetz durchzuführen, und ich habe allen Anfeindungen zum Trotz es in der Tat auch nıcht durchgeführt."

(Zitiert nach: Verhandlungen des Deutschen Bundestages, 1. Wahlperiode, Stenographische Berichte, S. 43 I I).

Die Fachbereichskonferenz ist sicher, würde ein Sozialarbeiter oder ein Lehrer eine solche Aussage wagen, er würde noch heute entlassen.

Die Fachbereichskonferenz weist schließlich darauf hin, daß es unter den Parteifreunden des Abg. Borsche einige gegeben hat und gibt, die immer dann auf Gesetz und Ordnung beharren, wenn diese Gesetze und diese Ordnung hinter den Anforderungen fortschrittlicher gesellschaftlicher Praxis zurückbleiben, daß sie aber schnell jedes Gesetz beiseite schieben, wenn die von ihnen für notwendig gehaltene Herstellung von Ruhe und Ordnung durch parlamentarisch legitimierte Gesetze erschwert wird.

Deutlich machen kann man dies an der Entwicklung von Prof. Theodor Maunz, 
bei dem der von der Anfrage betroffene Kollege während seines Studiums in München 1961/62 Staatsrecht hörte.

Prof. Maunz war damals gleichzeitig bayrischer Kultusminister. Neben seinem Kommentar zum Bonner Grundgesetz hat Prof. Maunz u. a. auch ein Buch über „Gestalt und Recht der Polizei« geschrieben. In diesem 1943 erschienenen Buch finden sich zur Bindung von Staatsbediensteten an das Gesetz u. a. folgende Ausführungen:

"Aus verständlichen Gründen wandte sıch die neue Polizei nach der Machtergreifung gegen das Einfangen ihrer Tätıgkeit in Normen, das unter dem Blickpunkt der geschıchtlichen Ereignisse als liberal erscheinen mußte und das die Durchschlagskraft des polizıelichen Wirkens hemmte. *

(Maunz a. a. O., S. 9)

"Zweck und Arbeitsumfang der Geheimen Staatspolizei nach dem heute erreichten Stand können in keiner Weise durch die gesetzlichen Bestimmungen erschöpft werden, die für sie erlassen worden sind. Das ist wohl letzten Endes der Grund dafür, daß eıne Reihe von geltenden Vorschriften nıcht mehr veröffentlicht worden ist. Zu ihnen gehören die Vorschriften über die polizeiliche Schutzhaft. Bekannt ist die rechtliche Ausgangsstellung für das Wirken der Geheimen Staatspolizei, es ist die Verordnung zum Schutze von Volk und Staat vom 28.2.33, die durch Aufhebung des die Unverletzlichkeit der Freiheit der Person verbürgenden Verfassungsartikels Schranken des früheren Rechtssystems wegräumte."

(Maunz a.a. O., S. 49)

Die Fachbereichskonferenz fragt den Abg. Borsche:

Hält er es für angebracht, daß ein Parteifreund in einem Land der Bundesrepublik Deutschland Kultusminister werden kann, der - wie die Leseproben erkennen lassen - offensichtlich unter Mißachtung jeder freiheitlich demokratischen Grundordnung die Konzentrationslager juristisch absegnete?

Wenn nein: Hat er Konsequenzen aus diesem Vorgang gezogen? Welche?

\section{Symposium über die Genese von Kriminalitätsnormen}

Der Arbeitskreis Junger Kriminologen veranstaltet vom 6.3. (abends) bis 9. 3. 1975 (mittags) im Zentrum für interdisziplinäre Forschung, Bielefeld, ein Symposium über die Setzung von Kriminalitätsnormen (Vorbereitung: Rüdiger Lautmann, Hans Haferkamp, Fritz Sack).

Die Vorschriften des StGB, des Nebenstrafrechts, der Prozeßordnungen u. a. bilden einen Teil der institutionellen Rahmenbedingungen im Prozeß der sozialen Kontrolle, insbesondere in der Kriminalisierung. Nach früher herrschender und noch heute viel vertretener Meinung wurden diese Normen als traglos gegeben hingenommen; der Prozeł ihrer Entstehung blieb unanalysiert. Das Defizit an kriminologischer Theorie auf diesem Teilgebiet ist dementsprechend beträchtlich.

Es wird vorgeschlagen, auf dem Symposium eine Bestandsaufnahme der vorliegenden Forschungsergebnisse, der offenen Fragen sowie der laufenden und geplanten Projekte zu erarbeiten. Dabei könnten beispielsweise diese Einzelprobleme angesprochen werden:

- Ablauf und Aushandlung von Normsetzung auf ministerieller und parlamentarischer Ebene; 